\title{
A PHASE TRANSITION IN A QUANTUM ANHARMONIC CRYSTAL
}

\author{
A. Kargol, Y. Kozitsky* \\ Instytut Matematyki UMCS, 20-031 Lublin, Poland \\ e-mail: akargol@golem.umcs.lublin.pl, jkozi@golem.umcs.lublin.pl
}

(Received November 7, 2007)

\begin{abstract}
A translation invariant system of interacting quantum anharmonic oscillators indexed by the elements of a simple cubic lattice $\mathbb{Z}^{d}$ is considered. The anharmonic potential is of a general type, which in particular means that it might have no symmetry. For this system, we prove that the global polarization (obtained in the thermodynamic limit) gets discontinuous at a certain value of the external field provided $d \geq 3$, and particle mass as well as interaction intensity are big enough. The proof is based on the representation of local Gibbs states of the model in terms of path measures and thereby on the use of the infrared estimates and the Garsia-Rodemich-Rumsey inequality.

Key words: theory of liquids, mixtures, statistical field theory, functional methods, collective variables.

PACS number(s): 63.20.Ry, 64.60.-i
\end{abstract}

Dedicated to Professor I. O. Vakarchuk on the occasion of his 60th birthday.

No single day without forty written pages! Vak's iron rule

\section{INTRODUCTION AND SETUP}

The theory of phase transitions in quantum systems has essential peculiarities, which distinguish it from the corresponding theory of classical systems. In this context, it suffices to mention that the existence of phase transitions in the three-dimensional isotropic quantum Heisenberg model has not been proven yet.

For lattice models, most of the results in this domain were obtained by means of quantum versions of the method of infrared bounds developed in [1]. The first publication in which the infrared estimates were applied to quantum spin models seems to be article [2]. After certain modifications this method had also been applied to a number of models with unbounded Hamiltonians [3-7], the main characteristic feature of which was the $Z_{2}$-symmetry broken by the phase transition. This symmetry allowed for obtaining an estimate crucial for the method. However in classical models, for proving phase transitions by means of the infrared estimates, symmetry was not especially important, see Theorem 3.5 in [1] and the discussion preceding this theorem. There might be two explanations of such a discrepancy: (a) the symmetry was the key element, but only of the methods employed therein, and, like in the classical case, its lack does not imply the lack of phase transitions; (b) the symmetry is crucial in view of e.g. quantum effects, which stabilize the system, see [8-10]. So far, there has been no possibility to check which of these explanations is true. In this article, we employ new tools based on recent advances in stochastic analysis and prove that the system of interacting quantum anharmonic oscillators without any symmetry undergoes a phase transition if in particular the oscillator mass is sufficiently big. In this case, the oscillators are 'close' to their classical analogs and hence quantum effects are not so strong, see [11]. As a consequence, the dilemma mentioned above has been solved in favor of explanation (a).

Infinite systems of interacting quantum anharmonic oscillators possess many interesting properties and applications. If the oscillators are indexed by the elements of a crystal lattice, the model is called (with a certain abuse of terminology) a quantum anharmonic crystal. Mostly such models are related with ionic crystals containing localized light particles oscillating in the field created by heavy ionic complexes. An example here can be a KDP-type ferroelectric with hydrogen bounds in which the particles are protons or deuterons performing onedimensional oscillations along the bounds. It is believed that structural phase transitions in such ferroelectrics are triggered by the ordering of the protons [12-14]. Thereby, quantum anharmonic crystals may have direct application to the theory of ferroelectrics with hydrogen bounds and the development of their rigorous theory remains a significant mathematical task.

To models of this kind the method of infrared estimates was first applied in [3,4], where the anharmonic potential was $Z_{2}$-symmetric and of $\phi^{4}$ type. These two properties allowed for obtaining the crucial estimate by means of a purely quantum tool - the Bogoliubov inequality. Afterwards in [5-7], the method of infrared estimates was extended to cover the case of $Z_{2}$-symmetric anharmonic potentials of the general type, which have two sufficiently deep wells. This was achieved by means 
of a representation of the Gibbs states in the form of imaginary-time Feynman path integrals. The approach in quantum statistical physics based on this representation is called Euclidean due to its conceptual similarity with the corresponding approach in quantum field theory, see $[15,16]$. In the Euclidean approach, the model is treated as a system of interacting classical spins, which are infinite-dimensional and unbounded. Due to this fact the method of [1] may be applied directly if the estimate mentioned above is obtained.

In this article, we prove the existence of phase transitions in quantum anharmonic crystals with possibly asymmetric anharmonic potentials. We employ an updated version of the Euclidean representation used in [5-7], combined with new techniques of the stochastic analysis. Namely, we prove an analog of Lemma 3.4 of [1] by means of the Garsia-Rodemich-Rumsey lemma, which then is used to prove an analog of Theorem 3.5 of [1].

A general version of the model we consider has the following heuristic Hamiltonian

$$
H=-\frac{1}{2} \sum_{\ell, \ell^{\prime}} J_{\ell \ell^{\prime}} q_{\ell} q_{\ell^{\prime}}+\sum_{\ell} H_{\ell}
$$

where the sums run through a crystal lattice $\mathbb{L}$. For simplicity we assume that $\mathbb{L}$ is a simple cubic lattice $\mathbb{Z}^{d}$, the interaction is of the nearest-neighbor type and $V_{\ell}$ is independent of $\ell$, i. e., the model is translation invariant. The displacement $q_{\ell}$ is a one-dimensional vector. The interaction term in (1.1) is of the dipole-dipole type. The Hamiltonian

$$
H_{\ell}=H_{\ell}^{\text {har }}+V\left(q_{\ell}\right) \stackrel{\text { def }}{=} \frac{1}{2 m} p_{\ell}^{2}+\frac{a}{2} q_{\ell}^{2}+V\left(q_{\ell}\right), \quad a>0
$$

describes an isolated anharmonic oscillator of mass $m$ and momentum $p_{\ell}$. The Hamiltonian $H_{\ell}^{\text {har }}$ corresponds to a quantum harmonic oscillator of rigidity $a$. We assume that $J_{\ell \ell^{\prime}}=J>0$ if and only if $\left|\ell-\ell^{\prime}\right|=1$, which in particular means that the model is ferroelectric. Regarding the anharmonic potential, we assume that it contains an external field $h \in \mathbb{R}$ in the form

$$
V(x)=V_{0}(x)-h x .
$$

Furthermore, $V_{0}$ is a continuous real-valued function, such that $V_{0}(0)=0$ and for all $x \in \mathbb{R}$,

$$
A_{V} x^{2 r}+B_{V} \leq V_{0}(x)
$$

with certain constants $r>1, A_{V}>0, B_{V} \in \mathbb{R}$. An example of $V$ to bear in mind is

$$
V(x)=\sum_{s=2}^{2 r} b_{\ell}^{(s)} x^{s}-h x .
$$

Thus, the heuristic Hamiltonian of the model we shall consider is

$$
H=-\frac{J}{2} \sum_{\ell, \ell^{\prime}:\left|\ell-\ell^{\prime}\right|=1} q_{\ell} q_{\ell^{\prime}}+\sum_{\ell}\left[H_{\ell}^{\mathrm{har}}+V\left(q_{\ell}\right)\right] .
$$

Like all objects of this kind, Hamiltonian (1.5) has no direct mathematical meaning and is 'represented' by local Hamiltonians corresponding to non-void finite subsets $\Lambda \subset \mathbb{L}$. To indicate this property of a given $\Lambda$ we write $\Lambda \Subset \mathbb{L}$; by $|\Lambda|$ we denote the number of elements. In the sequel, the adjective local will always mean the property of being related to a certain $\Lambda \Subset \mathbb{L}$, whereas global will refer to the whole lattice. If we say that something holds for all $\ell$, we mean it holds for all $\ell \in \mathbb{L}$; sums like $\sum_{\ell}$ mean $\sum_{\ell \in \mathbb{L}}$. By $(\cdot, \cdot)$ and $|\cdot|$ we denote a scalar product and the norm in the Euclidean space $\mathbb{R}^{d}$, respectively.

The set $\{\Lambda\}_{\Lambda \Subset \mathbb{L}}$ is countable; it is a net with the order defined by inclusion. A linearly ordered sequence of subsets $\Lambda \Subset \mathbb{L}$, which exhausts the lattice $\mathbb{L}$, will be called a cofinal sequence. The limit of a sequence of appropriate $A_{\Lambda}$ taken along such a cofinal sequence $\mathcal{L}$ will be denoted by $\lim _{\mathcal{L}} A_{\Lambda}$; we write $\lim _{\Lambda / \mathbb{L}} A_{\Lambda}$ if the limit is taken along the net $\{\Lambda\}_{\Lambda \Subset \mathbb{L}}$. The same notations will be used in the case of limsup and liminf.

The local Hamiltonians, $H_{\Lambda}$, are obtained by restricting the corresponding summations over $\ell$ and $\ell^{\prime}$ to this $\Lambda$. That is,

$$
H_{\Lambda}=-\frac{J}{2} \sum_{\ell, \ell^{\prime} \in \Lambda:\left|\ell-\ell^{\prime}\right|=1} q_{\ell} q_{\ell^{+}}+\sum_{\ell \in \Lambda}\left[H_{\ell}^{\mathrm{har}}+V\left(q_{\ell}\right)\right] .
$$

A special kind of $\Lambda \Subset \mathbb{L}$ is the box

$$
\Lambda=(-L, L]^{d} \bigcap \mathbb{L}, \quad L \in \mathbb{N}
$$

which can be turned into a torus by setting periodic conditions on its boundary. The same can be done by equipping $\Lambda$ with the periodic distance $\left|\ell-\ell^{\prime}\right|_{\Lambda}$, the definition of which is standard. By $\mathcal{L}_{\text {box }}$ we denote the set of all boxes, which obviously is a cofinal sequence.

For the box $\Lambda$, we set

$$
H_{\Lambda}^{\mathrm{per}}=-\frac{J}{2} \sum_{\ell, \ell^{\prime} \in \Lambda:\left|\ell-\ell^{\prime}\right|_{\Lambda}=1} q_{\ell} q_{\ell^{\prime}}+\sum_{\ell \in \Lambda}\left[H_{\ell}^{\mathrm{har}}+V\left(q_{\ell}\right)\right],
$$

that is the periodic local Hamiltonian. In the sequel, by writing expressions like $H_{\Lambda}^{\text {per }}$ we tacitly assume that the corresponding $\Lambda$ is a box. Due to property (1.4), both $H_{\Lambda}$ and $H_{\Lambda}^{\text {per }}$ are self-adjoint operators in the physical Hilbert space $\mathcal{H}_{\Lambda}=L^{2}\left(\mathbb{R}^{|\Lambda|}\right)$, such that for every $\beta>0$,

$$
\begin{gathered}
Z_{\Lambda} \stackrel{\text { def }}{=} \operatorname{trace}\left[\exp \left(-\beta H_{\Lambda}\right)\right]<\infty \\
Z_{\Lambda}^{\text {per }} \stackrel{\text { def }}{=} \operatorname{trace}\left[\exp \left(-\beta H_{\Lambda}^{\text {per }}\right)\right]<\infty
\end{gathered}
$$

Thus, one can define the corresponding local Gibbs states

$$
\begin{aligned}
\varrho_{\Lambda}(A) & =\operatorname{trace}\left[A \exp \left(-\beta H_{\Lambda}\right)\right] / Z_{\Lambda}, \quad A \in \mathfrak{C}_{\Lambda}, \\
\varrho_{\Lambda}^{\text {per }}(A) & =\operatorname{trace}\left[A \exp \left(-\beta H_{\Lambda}^{\text {per }}\right)\right] / Z_{\Lambda}^{\text {per }} .
\end{aligned}
$$




\section{A. KARGOL, YU. KOZITSKY}

Here $\mathfrak{C}_{\Lambda}$ is the local algebra of observables, which is the $C^{*}$-algebra of all bounded linear operators on $\mathcal{H}_{\Lambda}$. As the main source on the 'algebraic' aspects of our theory we mention monograph [17].

We study the dependence of the averages (1.10) on $J$, and $h$. Among them are local polarizations (magnetizations, in a ferromagnetic terminology), defined as follows

$$
\begin{aligned}
& M_{\Lambda}^{\text {per }}(J, h)=\varrho_{\Lambda}^{\text {per }}\left(q_{\ell}\right), \\
& M_{\Lambda}(J, h)=\frac{1}{|\Lambda|} \sum_{\ell \in \Lambda} \varrho_{\Lambda}\left(q_{\ell}\right), \quad \ell \in \Lambda .
\end{aligned}
$$

One observes that the periodic polarization is indepen- dent of $\ell$ in view of the corresponding invariance of the Hamiltonian (1.8). Since the thermodynamic properties of the model reveal themselves in the thermodynamic limit, let us look at the asymptotic behavior of the net $\left\{M_{\Lambda}(j, h)\right\}_{\Lambda \Subset \mathbb{L}}$ and of the sequence $\left\{M_{\Lambda}^{\text {per }}(j, h)\right\}_{\Lambda \in \mathcal{L}_{\text {box }}}$. Here we present some relevant facts, the proof of which will be discussed in the subsequent sections.

\section{Proposition I.1 Both $\left\{M_{\Lambda}(j, h)\right\}_{\Lambda \Subset \mathbb{L}}$ and $\left\{M_{\Lambda}^{\text {per }}(j, h)\right\}_{\Lambda \in \mathcal{L}_{\text {box }}}$ are bounded.}

In view of this fact, we define

$$
\begin{aligned}
& M_{+}(J, h)=\max \left\{\lim \sup _{\Lambda \nearrow \mathbb{L}} M_{\Lambda}(J, h) ; \lim \sup _{\Lambda \in \mathcal{L}_{\mathrm{box}}} M_{\Lambda}^{\mathrm{per}}(J, h)\right\}, \\
& M_{-}(J, h)=\min \left\{\lim \inf _{\Lambda \nearrow \mathbb{L}} M_{\Lambda}(J, h) ; \lim \inf _{\Lambda \in \mathcal{L}_{\mathrm{box}}} M_{\Lambda}^{\text {per }}(J, h)\right\} .
\end{aligned}
$$

In the following, by a denumerable set we mean a set which is void, finite, or countable. In the statements below, if we do not mention $\beta$ and $J$, we mean that the statement holds for all possible values of these parameters.

Proposition I.2 There exists a denumerable set $\mathcal{R} \subset \mathbb{R}$, such that, for $h \in \mathcal{R}^{c} \stackrel{\text { def }}{=} \mathbb{R} \backslash \mathcal{R}$,

$$
M_{-}(J, h)=M_{+}(J, h) \stackrel{\text { def }}{=} M(J, h) .
$$

The polarization $M(J, h)$, as a function of $h$, is nondecreasing on $\mathcal{R}^{c}$; it is continuous on each of its open connected components.

Note that by $\mathcal{R}$ we mean the smallest set with the properties stated.

Let us make some comments. By Proposition I.1, it follows that for a specific cofinal sequence $\mathcal{L}$, which may also be composed by boxes, the corresponding sequence of local polarizations has a limit, which belongs to the interval $\left[M_{-}(J, h), M_{+}(J, h)\right]$. By Proposition I.2, this interval shrinks into a point if $h \in \mathcal{R}^{c}$, which merely means that, at such $h$, there exists a (global) polarization independent of the sequence $\mathcal{L}$ along which the thermodynamic limit has been taken. This polarization is continuous on the interval $\left(a_{-}, a_{+}\right) \subset \mathcal{R}^{c}$, where $a_{ \pm}$are two consecutive elements of $\mathcal{R}$. At such $a_{ \pm}, M(J, h)$ is discontinuous. Since $M(J, h) \in\left[M_{-}(J, h), M_{+}(J, h)\right]$, the discontinuity is of the first kind. Therefore, at each $a \in \mathcal{R}$, such that both $(a-\epsilon, a),(a, a+\epsilon)$ are subsets of $\mathcal{R}^{c}$ for a certain $\epsilon>0$, one has

$$
\lim _{h \uparrow a} M(J, h)<\lim _{h \downarrow a} M(J, h) .
$$

At the same time, the set $\mathcal{R}^{c}$ may have empty interior; hence, the global polarization may be nowhere continuous.
Now we are ready to define the main notion of the paper.

Definition I.3 The model considered undergoes a phase transition (of first order) at certain $\beta, J$, and $h_{*}$ if the global polarization, as a function of $h$ at fixed $\beta$ and $J$, is discontinuous at this $h_{*}$.

Note that this definition agrees with the known one given by L. Landau. Remarkably, we do not suppose that the phase transition breaks any symmetry.

Recall that $\mathbb{L}=\mathbb{Z}^{d}$ and that $m$ is the particle mass. The result of the paper is contained in the following statement.

Theorem I.4 For every $d \geq 3$ and $\beta>0$, there exist $m_{*}>0$ and $J_{*}>0$ such that for every $m>m_{*}$ and $J>J_{*}$, there exists $h_{*} \in \mathbb{R}$, possibly dependent on $\beta, m$, and $J$, such that $M(J, h)$ gets discontinuous at $h_{*}$, i.e., the model undergoes a phase transition.

In the next section, we give the proof of this theorem based on a number of lemmas, which we prove by employing the representation of the local states (1.10) in terms of path measures. In Section III, we give a brief description of the corresponding approach - article [18] may serve as the main source of details. The proof of the lemmas is given in Section IV.

\section{PROOF OF THE THEOREM}

As the inverse temperature $\beta$ plays no role in our consideration, see the formulation of Theorem I.4, from now on we set $\beta=1$. We also set $\hbar=1$, which merely means 
that $m=m_{\mathrm{ph}} / \hbar^{2}$, where $m_{\mathrm{ph}}$ is the physical mass of the particle.

The proof of Theorem I.4 will be given at the end of this section. It is based on a number of preparatory statements - Propositions and Lemmas. The former ones will be either taken from other sources or discussed in the subsequent parts of the article. Some of them will be proved here. Lemmas, which are more important and nontrivial, will be proved in Section IV.

Keeping in mind the dependence of the partition functions (1.9) on the interaction intensity and the external field, we define

$p_{\Lambda}(J, h)=\log Z_{\Lambda} /|\Lambda| ; \quad p_{\Lambda}^{\mathrm{per}}(J, h)=\log Z_{\Lambda}^{\mathrm{per}} /|\Lambda|$.

We call these functions the pressure and the periodic pressure, respectively; up to the sign they coincide with the free energy density corresponding to the free and periodic conditions on the boundary of $\Lambda$. The properties of the pressure are described by the following statements. The first one can be obtained directly from definition (2.1).
Proposition II.1 Each $p_{\Lambda}(J, h), p_{\Lambda}^{\mathrm{per}}(J, h)$ is an infinitely differentiable function of $h \in \mathbb{R}$ and $J \in \mathbb{R}$. It is also a convex function of both these parameters.

One can easily verify that

$$
\frac{\partial}{\partial h} p_{\Lambda}(J, h)=M_{\Lambda}(J, h), \quad \frac{\partial}{\partial h} p_{\Lambda}^{\text {per }}(J, h)=M_{\Lambda}^{\text {per }}(J, h),
$$

and

$$
\begin{aligned}
\frac{\partial^{2}}{\partial h^{2}} p_{\Lambda}(J, h) & =\frac{1}{|\Lambda|} \sum_{\ell, \ell^{\prime} \in \Lambda} K_{\ell \ell^{\prime}}^{\Lambda}(0,0), \\
\frac{\partial^{2}}{\partial h^{2}} p_{\Lambda}^{\text {per }}(J, h) & =\frac{1}{|\Lambda|} \sum_{\ell, \ell^{\prime} \in \Lambda} K_{\ell \ell^{\prime}}^{\Lambda}(0,0 \mid \text { per }),
\end{aligned}
$$

where for $\tau, \tau^{\prime} \in[0,1], \tau \leq \tau^{\prime}$, we have set

$$
\begin{aligned}
& K_{\ell \ell^{\prime}}^{\Lambda}\left(\tau, \tau^{\prime}\right)=\Gamma_{\ell \ell^{\prime}}^{\Lambda}\left(\tau, \tau^{\prime}\right)-\varrho_{\Lambda}\left(q_{\ell}\right) \cdot \varrho_{\Lambda}\left(q_{\ell}\right) \\
& K_{\ell \ell^{\prime}}^{\Lambda}\left(\tau, \tau^{\prime} \mid \text { per }\right)=\Gamma_{\ell \ell^{\prime}}^{\Lambda}\left(\tau, \tau^{\prime} \mid \text { per }\right)-\varrho_{\Lambda}^{\text {per }}\left(q_{\ell}\right) \cdot \varrho_{\Lambda}^{\text {per }}\left(q_{\ell}\right),
\end{aligned}
$$

and

$$
\begin{aligned}
& \Gamma_{\ell \ell^{\prime}}^{\Lambda}\left(\tau, \tau^{\prime}\right)=\varrho_{\Lambda}\left\{q_{\ell} \exp \left[-\left(\tau^{\prime}-\tau\right) H_{\Lambda}\right] q_{\ell^{\prime}} \exp \left[\left(\tau^{\prime}-\tau\right) H_{\Lambda}\right]\right\} \\
& \Gamma_{\ell \ell^{\prime}}^{\Lambda}\left(\tau, \tau^{\prime} \mid \text { per }\right)=\varrho_{\Lambda}^{\text {per }}\left\{q_{\ell} \exp \left[-\left(\tau^{\prime}-\tau\right) H_{\Lambda}^{\text {per }}\right] q_{\ell^{\prime}} \exp \left[\left(\tau^{\prime}-\tau\right) H_{\Lambda}^{\text {per }}\right]\right\}
\end{aligned}
$$

Proposition II.2 It follows that for any $0 \leq \tau \leq \tau^{\prime} \leq 1$,

$$
K_{\ell \ell^{\prime}}^{\Lambda}\left(\tau, \tau^{\prime}\right) \geq 0, \quad K_{\ell \ell^{\prime}}^{\Lambda}\left(\tau, \tau^{\prime} \mid \text { per }\right) \geq 0 .
$$

The next statement clarifies the situation with the limiting pressure.

Proposition II.3 For every $J \geq 0$ and $h \in \mathbb{R}$,

$$
\lim _{\Lambda \succ \mathbb{L}} p_{\Lambda}(J, h)=\lim _{\mathcal{L}_{\mathrm{box}}} p_{\Lambda}^{\text {per }}(J, h) \stackrel{\text { def }}{=} p(J, h) .
$$

Thereby, the limiting pressure always exists and is independent of the way the limit has been taken. This fact together with those established by Proposition II.1 yield some important information regarding the properties of the global polarizations.

We recall that for the function $\varphi: \mathbb{R} \rightarrow \mathbb{R}$, the onesided derivatives are

$$
\varphi_{ \pm}^{\prime}(t)=\lim _{\epsilon \downarrow 0} \frac{\varphi(t \pm \epsilon)-\varphi(t)}{ \pm \epsilon} .
$$

It is known, see e.g., [25], pages 34-37, that

Proposition II.4 For a convex function $\varphi: \mathbb{R} \rightarrow \mathbb{R}$, (a) the one-sided derivatives $\varphi_{ \pm}^{\prime}(t)$ exist for every $t \in \mathbb{R} ;$ the set $\left\{t \in \mathbb{R} \mid \varphi_{+}^{\prime}(t) \neq \varphi_{-}^{\prime}(t)\right\}$ is denumerable;

(b) the point-wise limit $\varphi$ of a sequence of convex functions $\left\{\varphi_{n}\right\}_{n \in \mathbb{N}}$ is a convex function; if $\varphi$ and all $\varphi_{n}$ 's are differentiable at the given $t$, the sequence $\left\{\varphi_{n}^{\prime}(t)\right\}_{n \in \mathbb{N}}$ converges to $\varphi^{\prime}(t)$.

The proof of Proposition I.2 readily follows from the convexity of the pressure and the latter statement.

Next we introduce the so called Duhamel two-point function - a traditional element of the theory of phase transitions in quantum systems, see [2]. In our context, it is

$$
\begin{aligned}
D_{\ell \ell^{\prime}}^{\Lambda} & =\int_{0}^{1} \int_{0}^{1} \Gamma_{\ell \ell^{\prime}}^{\Lambda}\left(\tau, \tau^{\prime} \mid \text { per }\right) \mathrm{d} \tau \mathrm{d} \tau^{\prime} \\
& =\int_{0}^{1} \Gamma_{\ell \ell^{\prime}}^{\Lambda}(0, \tau \mid \text { per }) \mathrm{d} \tau, \quad \ell, \ell^{\prime} \in \Lambda .
\end{aligned}
$$

As follows from (2.8) and (2.6),

$$
D_{\ell \ell^{\prime}}^{\Lambda} \geq 0
$$

for all boxes $\Lambda$ and $\ell, \ell^{\prime}$. By construction, $D_{\ell \ell^{\prime}}^{\Lambda}$ is invariant with respect to the translations of the torus $\Lambda$. To employ this property we introduce the set $\Lambda_{*}$ (a Brillouin zone) consisting of 


$$
\begin{aligned}
& p=\left(p_{1}, \ldots, p_{d}\right), \quad p_{j}=-\pi+\frac{2 \pi}{L} s_{j}, \\
& s_{j}=1, \ldots, 2 L, j=1, \ldots, d,
\end{aligned}
$$

and thereby the Fourier transformation

$$
\begin{aligned}
\widehat{D}_{p}^{\Lambda} & =\sum_{\ell^{\prime} \in \Lambda} D_{\ell \ell^{\prime}}^{\Lambda} \exp \left[\imath\left(p, \ell-\ell^{\prime}\right)\right], \quad p \in \Lambda_{*}, \\
D_{\ell \ell^{\prime}}^{\Lambda} & =\frac{1}{|\Lambda|} \sum_{p \in \Lambda_{*}} \widehat{D}_{p}^{\Lambda} \exp \left[-\imath\left(p, \ell-\ell^{\prime}\right)\right] .
\end{aligned}
$$

Now we need to make a more formal definition of the thermodynamic limit, c.f. Proposition I.1. Recall that for a given $\Lambda \Subset \mathbb{L}, \mathfrak{C}_{\Lambda}$ is the algebra of local observables, being the $C^{*}$-algebra of all bounded linear operators on the physical Hilbert space $\mathcal{H}_{\Lambda}$. For $\Lambda \subset \Lambda^{\prime}$, one can define the canonical embedding $\mathfrak{C}_{\Lambda} \hookrightarrow \mathfrak{C}_{\Lambda^{\prime}}$, up to which $\mathfrak{C}_{\Lambda}$ is a subalgebra of $\mathfrak{C}_{\Lambda^{\prime}}$. Thereby, we define the algebra of all local observables

$$
\mathfrak{C}^{\text {loc }}=\bigcup_{\Lambda \Subset \mathbb{L}} \mathfrak{C}_{\Lambda}
$$

Equipped with the norm inherited from $\mathfrak{C}_{\Lambda}$ it becomes a normed space, although not complete. Its completion, $\mathfrak{C}$, is called the algebra of quasi-local observables. It is also a $C^{*}$-algebra. One can show that the union in (2.12) may be taken over any cofinal sequence $\mathcal{L}$.

The local states (1.10) describe the properties of a portion of the system, while the whole system is described by the states defined on the algebra $\mathfrak{C}$.

Proposition II.5 For every $J>0$ and $h \in \mathbb{R}$, there exists a tendenly to infinity sequence $\left\{L_{n}\right\}_{n \in \mathbb{N}}$ and hence sequence $\left\{\Lambda_{n}\right\}_{n \in \mathbb{N}}$ of the corresponding boxes (1.7), such that sequence $\left\{\varrho_{\Lambda_{n}}^{\text {per }}\right\}_{n \in \mathbb{N}}$ of the states (1.10) converges to a translation invariant state $\varrho^{\mathrm{per}}$ on the algebra $\mathfrak{C}$ (called a periodic state) in such a way that for every polynomial $P\left(q_{\Delta}\right), \Delta \Subset \mathbb{L}, q_{\Delta}=\left(q_{\ell}\right)_{\ell \in \Delta}$,

$$
\lim _{n \rightarrow+\infty} \varrho_{\Lambda_{n}}^{\text {per }}\left[P\left(q_{\Delta}\right)\right]=\varrho^{\text {per }}\left[P\left(q_{\Delta}\right)\right] .
$$

Furthermore, this convergence also yields that for every $\ell, \ell^{\prime}$,

$$
\Gamma_{\ell \ell^{\prime}}^{\Lambda_{n}}(\cdot, \cdot \mid \text { per }) \rightarrow \Gamma_{\ell \ell^{\prime}}(\cdot, \cdot \mid \text { per }),
$$

uniformly on $\tau, \tau^{\prime} \in[0,1]$.

Note that by (2.13), one has, c.f. (1.12),

$$
\varrho^{\text {per }}\left(q_{\ell}\right)=\lim _{n \rightarrow+\infty} M_{\Lambda_{n}}^{\text {per }}(J, h) .
$$

In view of (2.14), we set, c.f. (2.8),

$$
D_{\ell \ell^{\prime}}=\int_{0}^{1} \Gamma_{\ell \ell^{\prime}}(0, \tau \mid \text { per }) \mathrm{d} \tau \text {. }
$$

By construction, $\Gamma_{\ell \ell^{\prime}}(\cdot, \cdot \mid$ per $)$, and hence $D_{\ell \ell^{\prime}}$, are invariant with respect to the translations of $\mathbb{Z}^{d}$. Thus, one can define

$$
\begin{aligned}
\widehat{D}_{p} & =\sum_{\ell^{\prime}} D_{\ell \ell^{\prime}} \exp \left[\imath\left(p, \ell-\ell^{\prime}\right)\right], \quad p \in(-\pi, \pi]^{d}, \\
D_{\ell \ell^{\prime}} & =\frac{1}{(2 \pi)^{d}} \int_{(\pi, \pi]^{d}} \widehat{D}_{p} \exp \left[-\imath\left(p, \ell-\ell^{\prime}\right)\right] \mathrm{d} p .
\end{aligned}
$$

Proposition II.6 Suppose that for a given $J>0$, there exists $h_{*}$ such that

$$
\varrho^{\text {per }}\left(q_{\ell}\right)=0
$$

at $h=h_{*}$. Suppose in addition that at $h=h_{*}$ there exists a sequence of boxes $\left\{\Lambda_{k}\right\}_{k \in \mathbb{N}}$ such that

$$
\lim _{k \rightarrow+\infty} \frac{1}{\left|\Lambda_{k}\right|} \sum_{\ell^{\prime} \in \Lambda_{k}} D_{\ell \ell^{\prime}}=\lim _{k \rightarrow+\infty} \frac{1}{\left|\Lambda_{k}\right|^{2}} \sum_{\ell, \ell^{\prime} \in \Lambda_{k}} D_{\ell \ell^{\prime}}>0 .
$$

Then the model undergoes the phase transition at these $J$ and $h_{*}$.

By (2.17) and (2.9) it follows that

$$
\widehat{D}_{0} \geq \sum_{\ell^{\prime} \in \Lambda_{k}} D_{\ell \ell^{\prime}}
$$

for any $\Lambda_{k}$ mentioned in the above statement. Thus, if (2.19) holds, then there exists $c>0$ such that

$$
\widehat{D}_{0} \geq c\left|\Lambda_{k}\right|, \quad \text { for any } k \in \mathbb{N} \text {. }
$$

This means that $\widehat{D}_{p}$ is singular at $p=0$ in this case. On the other hand, by the second line of (2.17) this infrared singularity is integrable; hence, $\widehat{D}_{p}$ is a distribution. Thus, one can write it in the form

$$
\widehat{D}_{p}=(2 \pi)^{d} \varkappa \delta(p)+g(p),
$$

where $\delta$ is the Dirac $\delta$-function and $g(p)$ is regular at $p=0$. By $(2.9), g(p)$, for all $p$, and $\varkappa$ are nonnegative; $\varkappa$ is positive if (2.19) holds. Note that (2.21) is similar to the Källen-Lehmann representation known in quantum field theory. For more on this subject, see Introduction in [1].

By (2.17) and (2.21),

$$
\varkappa=D_{\ell \ell}-\frac{1}{(2 \pi)^{d}} \int_{(-\pi, \pi]^{d}} g(p) \mathrm{d} p
$$

hence, in order to prove that $\varkappa>0$ one has to estimate $D_{\ell \ell}$ from below and $g(p)$ from above. The estimate of $g(p)$ is obtained in the next statement which we prove by means of a method used in [2], see Example 4, pages 362-364.

Proposition II.7 Suppose there exists a continuous $b$ : $(-\pi, \pi]^{d} \backslash\{0\} \rightarrow[0,+\infty)$ satisfying the condition

$$
\int_{(-\pi, \pi]^{d}} b(p) \mathrm{d} p<\infty
$$

and such that for all boxes $\Lambda$,

$$
\widehat{D}_{p}^{\Lambda} \leq b(p), \quad \text { for all } p \in \Lambda_{*} \backslash\{0\} .
$$

Then the function $g$ in representation (2.21) obeys the estimate

$$
g(p) \leq b(p), \quad \text { for all } p \in(-\pi, \pi]^{d} \backslash\{0\} .
$$


Proof: Let $\psi: \mathbb{Z}^{d} \rightarrow \mathbb{C}$ be such that there exists $\Delta \Subset \mathbb{L}$, for which $\psi_{\ell}=0$ for all $\ell \in \Delta^{c}=\mathbb{L} \backslash \Delta$. We also assume that

$$
\sum_{\ell \in \Delta} \psi_{\ell}=0
$$

Pick up the box $\Lambda$, such that $\Delta \subset \Lambda$. For this $\Lambda$, we set $\Psi^{\Lambda}=\sum_{\ell, \ell^{\prime} \in \Lambda} D_{\ell \ell^{\prime}}^{\Lambda} \psi_{\ell} \bar{\psi}_{\ell^{\prime}}=\frac{1}{|\Lambda|} \sum_{p \in \Lambda_{*} \backslash\{0\}} \widehat{D}_{p}^{\Lambda}|\hat{\psi}(p)|^{2}$,

where

$$
\hat{\psi}(p)=\sum_{\ell \in \Lambda} \psi_{\ell} \exp [\imath(p, \ell)]=\sum_{\ell \in \Delta} \psi_{\ell} \exp [\imath(p, \ell)]
$$

which can obviously be defined for all $p \in(-\pi, \pi]^{d}$. The $p=0$ term in the sum in (2.27) has been excluded in view of (2.26). Then by (2.24) we have

$$
\lim _{L \rightarrow+\infty} \Psi^{\Lambda_{L}} \leq \frac{1}{(2 \pi)^{d}} \int_{(-\pi, \pi]^{d}}|\hat{\psi}(p)|^{2} b(p) \mathrm{d} p .
$$

In view of (2.28) and the bound (2.24), the limit on the left-hand side exists by Lebesgue's dominated convergence theorem, at least for a subsequence $\left\{L_{n}\right\}$. Then

$$
\begin{aligned}
& \frac{1}{(2 \pi)^{d}} \int_{(-\pi, \pi]^{d}} \widehat{D}_{p}|\hat{\psi}(p)|^{2} \mathrm{~d} p=\lim _{L_{n} \rightarrow+\infty} \Psi^{\Lambda_{L_{n}}} \\
& \leq \frac{1}{(2 \pi)^{d}} \int_{(-\pi, \pi]^{d}}|\hat{\psi}(p)|^{2} b(p) \mathrm{d} p .
\end{aligned}
$$

For every $p_{*} \neq 0$, one can choose the function $\psi$ in such a way that $|\hat{\psi}(p)|^{2}$ is close to the $\delta$-function concentrated at this $p_{*}$. This yields (2.25).

As was mentioned above, the proof of the theorem is based on the infrared estimates. A detailed presentation of this method in its application to quantum anharmonic crystals can be found in $[5-7,18]$. By means of it we make estimate (2.24) precise.

Proposition II.8 For every box $\Lambda$, and any $p \in \Lambda_{*} \backslash\{0\}$,

$$
0<\widehat{D}_{p}^{\Lambda} \leq 1 / J E(p),
$$

where

$$
E(p)=\sum_{j=1}^{d}\left[1-\cos p_{j}\right]
$$

Note that the function $1 / E(p)$ is integrable on $(-\pi, \pi]^{d}$ for $d \geq 3$.

Now we give the statements which finalize the preparation of the proof of the theorem.

Lemma II.9 For every $m_{0}>0$, there exist $h_{ \pm}\left(m_{0}\right) \in$ $\mathbb{R}, h_{+}\left(m_{0}\right)>h_{-}\left(m_{0}\right)$, such that for all $m>m_{0}$ and $J \geq 0$,

$$
\begin{aligned}
& M_{\Lambda}^{\text {per }}(J, h)>0, \quad \text { for all } h>h_{+}\left(m_{0}\right) \\
& M_{\Lambda}^{\text {per }}(J, h)<0, \quad \text { for all } h<h_{-}\left(m_{0}\right) .
\end{aligned}
$$

The next statement is an analog of Lemma 3.4 of [1].

Lemma II.10 There exist positive numbers $\varepsilon, \delta$, and $m_{*}>m_{0}$, where $m_{0}$ is as in Proposition II.9, such that for all $\Lambda$ and $m>m_{*}$,

$$
p_{\Lambda}^{\mathrm{per}}(J, h)-p_{\Lambda}^{\mathrm{per}}(0, h) \geq d(\varepsilon J-\delta) .
$$

Lemma II.11 Let $m_{*}$ be as above. Then for every $\Lambda$, $m>m_{*}, J>0$, and $h \in \mathbb{R}$,

$$
\varrho_{\Lambda}^{\text {per }}\left(q_{\ell}^{2}\right) \geq\left[p_{\Lambda}^{\text {per }}(J, h)-p_{\Lambda}^{\text {per }}(0, h)\right] / J d .
$$

One observes that $p_{\Lambda}^{\text {per }}(0, h)$ does not depend on $\Lambda$. By means of Lemmas II.10, II.11 we have

$$
\varrho_{\Lambda}^{\mathrm{per}}\left(q_{\ell}^{2}\right) \geq \varepsilon-\delta / J
$$

which can be used to estimate $D_{\ell \ell}$ from below, see (2.22). To this end we use the Bruch-Falk inequality, see Theorem 3.1 in [2] or Theorem IV.7.5, page 392 of [25]. Recall that by (2.8) and (2.5),

$$
D_{\ell \ell}^{\Lambda}=\int_{0}^{1} \varrho_{\Lambda}^{\mathrm{per}}\left\{q_{\ell} \exp \left[-\tau H_{\Lambda}^{\mathrm{per}}\right] q_{\ell} \exp \left[-\tau H_{\Lambda}^{\mathrm{per}}\right]\right\} \mathrm{d} \tau
$$

\section{Proposition II.12 (Bruch-Falk Inequality)}

It follows that

$$
D_{\ell \ell}^{\Lambda} \geq \varrho_{\Lambda}^{\mathrm{per}}\left(q_{\ell}^{2}\right) \cdot f\left(\frac{1}{4 m \varrho_{\Lambda}^{\mathrm{per}}\left(q_{\ell}^{2}\right)}\right)
$$

where the function $f:[0,+\infty) \rightarrow[0,1]$ is defined implicitly by

$$
f(\xi \tanh \xi)=\xi^{-1} \tanh \xi, \quad \text { for } \quad \xi>0 ; \quad \text { and } \quad f(0)=1 \text {. }
$$

The function $f$ is differentiable, convex and monotone decreasing on $(0,+\infty)$, and such that

$$
\lim _{\xi \rightarrow+\infty} \xi f(\xi)=1
$$

see Theorem A.2 in [2]. As the right-hand side of (2.36) is independent of $\Lambda$, one can pass here to the thermodynamic limit along the same sequence of boxes as in (2.15). Then

$$
D_{\ell \ell} \geq(\varepsilon-\delta / J) \theta(J) ; \quad \theta(J) \stackrel{\text { def }}{=} f\left(J / 4 m_{*}(\varepsilon J-\delta)\right),
$$

where $m_{*}$ is the same as in Lemma II.10 and $J>\delta / \varepsilon$.

Proof of Theorem I.4: Suppose the model has no phase transitions. Then by Proposition I.2 the set $\mathcal{R}$ is 


\section{A. KARGOL, YU. KOZITSKY}

void and the polarization $M(J, h)$ is a continuous function of $h \in \mathbb{R}$ for each $J>0$. This yields, see (1.13) and Lemma II.9, that for every $J>0$, there exists $h_{*} \in \mathbb{R}$, possibly depending on $J$, such that $M\left(J, h_{*}\right)=0$. On the other hand, by (2.39) and (2.30), (2.25), we have in $(2.22)$

$$
\varkappa \geq(\varepsilon-\delta / J) \theta(J)-\Phi(d) / J,
$$

where

$$
\Phi(d) \stackrel{\text { def }}{=} \frac{1}{(2 \pi)^{d}} \int_{(-\pi, \pi]^{d}} \frac{\mathrm{d} p}{E(p)},
$$

which exists for $d \geq 3$, see (2.31). Note that the righthand side of (2.40) does not depend on $h$. Now we pick up $J_{*}>0$ such that $\varkappa>0$ for all $J>J_{*}$. For such $J$ and $m>m_{*}, D_{\ell \ell^{\prime}}$ does not tend to zero as $\left|\ell-\ell^{\prime}\right| \rightarrow+\infty$, see (2.17); hence, (2.19) holds. Then by Lemma II.6, we get a contradiction with the supposition made at the beginning of the proof.

\section{THE EUCLIDEAN APPROACH}

States (1.10) define the thermodynamic properties of the portion of the system located in $\Lambda$. The dynamics of this portion is described by the time automorphisms

$$
\begin{aligned}
& A \mapsto \mathfrak{a}_{t}^{\Lambda}(A)=\exp \left(\imath t H_{\Lambda}\right) A \exp \left(-\imath t H_{\Lambda}\right), \\
& t \in \mathbb{R}, \quad \Lambda \Subset \mathbb{L} .
\end{aligned}
$$

As both dynamics and thermodynamics are determined by the same object - the local Hamiltonian $H_{\Lambda}-$ the states (1.10) obey the KMS condition, see e. g. [20].

Let $\mathfrak{M}_{\Lambda}^{\text {cont }}$ be the algebra of all multiplication operators by bounded continuous functions $F \in C_{\mathrm{b}}\left(\mathbb{R}^{\nu|\Lambda|}\right)$. One can prove, see [21], that the linear span of the operators $\mathfrak{a}_{t_{1}}^{\Lambda}\left(F_{1}\right) \cdots \mathfrak{a}_{t_{n}}^{\Lambda}\left(F_{n}\right)$ with all possible choices of $n \in \mathbb{N}$, $t_{1}, \ldots, t_{n} \in \mathbb{R}$, and $F_{1}, \ldots, F_{n} \in \mathfrak{M}_{\Lambda}^{\text {cont }}$, is dense in $\mathfrak{C}_{\Lambda}$ in the topology in which the states (1.10) are continuous. Therefore, such states are fully determined by their values on the mentioned products, i.e., by the local Green functions

$$
G_{F_{1}, \ldots, F_{n}}^{\Lambda}\left(t_{1}, \ldots, t_{n}\right)=\varrho_{\Lambda}\left[\mathfrak{a}_{t_{1}}^{\Lambda}\left(F_{1}\right) \cdots \mathfrak{a}_{t_{n}}^{\Lambda}\left(F_{n}\right)\right]
$$

and the corresponding periodic local Green functions. They can be looked upon, see $[18,22,23]$, as the restrictions of functions $G_{F_{1}, \ldots, F_{n}}^{\Lambda}$ analytic on the domains

$$
\mathcal{D}_{\beta}^{n}=\left\{\left(t_{1}, \ldots, t_{n}\right) \in \mathbb{C}^{n} \mid 0<\Im\left(t_{1}\right)<\cdots<\Im\left(t_{n}\right)<\beta\right\}
$$

and continuous on their closures $\overline{\mathcal{D}_{\beta}^{n}}$. For every $n \in \mathbb{N}$, the subset

$$
\left\{\left(t_{1}, \ldots, t_{n}\right) \in \mathcal{D}_{\beta}^{n} \mid \Re\left(t_{1}\right)=\ldots=\Re\left(t_{n}\right)=0\right\},
$$

is an inner set of uniqueness for functions analytic in $\mathcal{D}_{\beta}^{n}$, see [24], pages 101 and 325. This means that the Matsubara functions

$$
\Gamma_{F_{1}, \ldots, F_{n}}^{\Lambda}\left(\tau_{1}, \ldots, \tau_{n}\right) \stackrel{\text { def }}{=} G_{F_{1}, \ldots, F_{n}}^{\Lambda}\left(\imath \tau_{1}, \ldots, \imath \tau_{n}\right),
$$

defined for $0 \leq \tau_{1} \leq \cdots \leq \tau_{n} \leq \beta$, uniquely determine the corresponding Green functions and hence the states $\varrho_{\Lambda}$. They have the property

$$
\Gamma_{F_{1}, \ldots, F_{n}}^{\Lambda}\left(\tau_{1}+\vartheta, \ldots, \tau_{n}+\vartheta\right)=\Gamma_{F_{1}, \ldots, F_{n}}^{\Lambda}\left(\tau_{1}, \ldots, \tau_{n}\right),
$$

where addition is of modulo $\beta$. This periodicity and the analyticity of the Green functions imply the KMS property of the state $\varrho_{\Lambda}[20,22,23]$.

This passage from real to imaginary values of time, see (3.4), is conceptually similar with the Euclidean approach in quantum field theory, see e.g., [15], It allows for employing the properties of the semi-groups of operators $\exp \left(-\tau H_{\Lambda}\right)$, where $\tau$ varies in the interval $[0, \beta]$ with the periodic condition at the endpoints. This semigroup generates a $\beta$-periodic Markov process, see [20]. Its canonical realization is described by a probability measure on a space of continuous paths. Thereby, the
Matsubara functions (3.5) can be written in the form of integrals taken with respect to this measure, which opens the possibility to apply here powerfull methods from the stochastic analysis and measure theory. Our proof will be based on some of them.

Now let us introduce the mentioned path spaces and measures. For simplicity, we perform this for $\beta=1$. In view of the periodicity at the endpoints of the interval $[0,1]$ we associate it with the circle $S$ of unit length, considered as a Riemannian manifold equipped with the Lebesque measure $\mathrm{d} \tau$. Therefore, each such path can be considered as a classical spin at $\ell$, though infinitedimensional. It takes values in the space $\mathcal{C}$ of all continuous functions $\phi: S \rightarrow \mathbb{R}$, which can also be considered as continuous functions $[0,1] \rightarrow \mathbb{R}$ taking equal values at the endpoints. We equip $\mathcal{C}$ with the usual supremum norm $|\cdot|_{\mathcal{C}}$, in which is becomes a Banach space. Along with $\mathcal{C}$ we use also spaces of Hölder-continuous functions $\phi: S \rightarrow \mathbb{R}$. For such a function and $\sigma \in(0,1 / 2)$, we set

$$
|\phi|_{\mathcal{C}_{\sigma}}=|\phi|_{\mathcal{C}}+\sup _{\tau, \tau^{\prime} \in S, \tau \neq \tau^{\prime}} \frac{\left|\phi(\tau)-\phi\left(\tau^{\prime}\right)\right|}{\left|\tau-\tau^{\prime}\right|_{S}^{\sigma}},
$$

where

$$
\left|\tau-\tau^{\prime}\right|_{S} \stackrel{\text { def }}{=} \min \left\{\left|\tau-\tau^{\prime}\right| ; 1-\left|\tau-\tau^{\prime}\right|\right\} .
$$

Then we set

$$
\mathcal{C}_{\sigma}=\left\{\left.\phi \in \mathcal{C}|| \phi\right|_{\mathcal{C}_{\sigma}}<\infty\right\}
$$




\section{A PHASE TRANSITION IN A QUANTUM ANHARMONIC CRYSTAL}

Equipped with norm (3.6), $\mathcal{C}_{\sigma}$ becomes a Banach space as well. Along with these spaces we shall use also the real Hilbert space $\mathcal{L}^{2} \stackrel{\text { def }}{=} L^{2}(S, \mathrm{~d} \tau)$, the scalar product and norm of which are denoted by $(\cdot, \cdot)_{\mathcal{L}^{2}}$ and $|\cdot|_{\mathcal{L}^{2}}$, respectively.
For $\Lambda \Subset \mathbb{L}$, we set

$\Omega_{\Lambda}=\left\{\omega_{\Lambda}=\left(\omega_{\ell}\right)_{\ell \in \Lambda} \mid \omega_{\ell} \in \mathcal{C}\right.$, for all $\left.\ell \in \Lambda\right\}$.

Thus, the representation of function (3.5) in the form of the integral mentioned above is, c.f. (2.5),

$$
\begin{aligned}
\Gamma_{F_{1}, \ldots, F_{n}}^{\Lambda}\left(\tau_{1}, \ldots, \tau_{n}\right) & =\operatorname{trace}\left[F_{1} \exp \left(-\left(\tau_{2}-\tau_{1}\right) H_{\Lambda}\right) \cdots F_{n-1} \exp \left(-\left(\tau_{n}-\tau_{n-1}\right) H_{\Lambda}\right) F_{n} \exp \left(-\left(1-\tau_{n}+\tau_{1}\right) H_{\Lambda}\right)\right] / Z_{\Lambda} \\
& =\int_{\Omega} F_{1}\left(\omega_{\Lambda}\left(\tau_{1}\right)\right) \cdots F_{n}\left(\omega_{\Lambda}\left(\tau_{n}\right)\right) \mu_{\Lambda}\left(\mathrm{d} \omega_{\Lambda}\right),
\end{aligned}
$$

where $\mu_{\Lambda}$ is the above-mentioned path probability measure, which we are going to construct now.

The Hamiltonian $H_{\ell}^{\text {har }}$, see $(1.2)$, generates a Gaussian periodic Markov process, known as the OrnsteinUhlenbeck velocity process. Its canonical realization on $\mathcal{C}$ is described by the path measure which one introduces as follows. In $\mathcal{L}^{2}$, we define the self-adjoint (LaplaceBeltrami type) operator

$$
A=-m \frac{\mathrm{d}^{2}}{\mathrm{~d} \tau^{2}}+a .
$$

Its spectrum consists of the eigenvalues

$$
\lambda_{k}=m(2 \pi k / \beta)^{2}+a, \quad k \in \mathbb{Z} .
$$

Thus, the inverse $A^{-1}$ is of trace class and the Fourier transform

$$
\begin{gathered}
\int_{L_{\beta}^{2}} \exp \left[\imath(\phi, v)_{\left.\mathcal{L}^{2}\right] \chi(\mathrm{d} v)}=\exp \left\{-\frac{1}{2}\left(A^{-1} \phi, \phi\right)_{L_{\beta}^{2}}\right\},\right. \\
\phi \in L_{\beta}^{2}
\end{gathered}
$$

defines a Gaussian measure $\chi$ on $\mathcal{L}^{2}$. Employing eigenvalues (3.12) one can show that for any $k \in \mathbb{N}$,

$$
\int_{\mathcal{L}^{2}}\left[\omega(\tau)-\omega\left(\tau^{\prime}\right)\right]^{2 k} \chi(d \omega) \leq \frac{2^{k} \Gamma(1 / 2+k)}{m^{k} \Gamma(1 / 2)} \cdot\left|\tau-\tau^{\prime}\right|_{\mathrm{per}}^{k},
$$

which by Kolmogorov's lemma, page 43 of [26], yields that

$$
\chi\left(\mathcal{C}^{\sigma}\right)=1, \quad \text { for all } \sigma \in(0,1 / 2) .
$$

Then $\chi\left(C_{\beta}\right)=1$ and we redefine $\chi$ as a probability measure on $\mathcal{C}$. An account of the properties of $\chi$ may be found in [18].

The measure $\chi$ is the local Euclidean Gibbs measure for a single harmonic oscillator. The measure $\mu_{\Lambda}$ which corresponds to a portion of interacting anharmonic oscillators located in $\Lambda \Subset \mathbb{L}$ is associated with a stationary periodic Markov process defined as follows. The marginal distributions of $\mu_{\Lambda}$ are given by the integral kernels of the operators $\exp \left(-\tau H_{\Lambda}\right), \tau \in[0,1]$. This means that

$\operatorname{trace}\left[F_{1} e^{-\left(\tau_{2}-\tau_{1}\right) H_{\Lambda}} F_{2} e^{-\left(\tau_{3}-\tau_{2}\right) H_{\Lambda}} \cdots F_{n} e^{-\left(\tau_{n+1}-\tau_{n}\right) H_{\Lambda}}\right] / \operatorname{trace}\left[e^{-H_{\Lambda}}\right]=\int_{\Omega_{\Lambda}} F_{1}\left(\omega_{\Lambda}\left(\tau_{1}\right) \cdots F_{n}\left(\omega_{\Lambda}\left(\tau_{n}\right)\right) \mu_{\Lambda}\left(\mathrm{d} \omega_{\Lambda}\right)\right.$

for all $F_{1}, \ldots, F_{n} \in L^{\infty}\left(\mathbb{R}^{|\Lambda|}\right), n \in \mathbb{N}$ and $\tau_{1}, \ldots, \tau_{n} \in S_{\beta}$, such that $\tau_{1} \leq \cdots \leq \tau_{n} \leq 1, \tau_{n+1}=\tau_{1}+1$. And vice verse, representation (3.16) uniquely, up to equivalence, defines $H_{\Lambda}$ (see [20]). In the same way, one obtains the connection between the periodic Hamiltonian (1.8) and the measure $\mu_{\Lambda}^{\mathrm{per}}$. By means of the Feynman-Kac formula $\mu_{\Lambda}^{\text {per }}$ is obtained as a Gibbs modification

$$
\mu_{\Lambda}^{\text {per }}\left(\mathrm{d} \omega_{\Lambda}\right)=\exp \left\{-I_{\Lambda}^{\text {per }}\left(\omega_{\Lambda}\right)\right\} \chi_{\Lambda}\left(\mathrm{d} \omega_{\Lambda}\right) / Z_{\Lambda},
$$

of the 'free measure'

$$
\chi_{\Lambda}\left(\mathrm{d} \omega_{\Lambda}\right)=\prod_{\ell \in \Lambda} \chi\left(\mathrm{d} \omega_{\ell}\right) .
$$

Here

$$
\begin{aligned}
I_{\Lambda}^{\mathrm{per}}\left(\omega_{\Lambda}\right) & =-\frac{J}{2} \sum_{\ell, \ell^{\prime} \in \Lambda:\left|\ell-\ell^{\prime}\right|_{\Lambda}=1}\left(\omega_{\ell}, \omega_{\ell^{\prime}}\right)_{\mathcal{L}^{2}} \\
& +\sum_{\ell \in \Lambda} \int_{0}^{1} V_{\ell}\left(\omega_{\ell}(\tau)\right) \mathrm{d} \tau
\end{aligned}
$$

is the energy functional describing the system of interacting paths $\omega_{\ell}$ located in the torus $\Lambda$, whereas

$$
Z_{\Lambda}^{\mathrm{per}}=\int_{\Omega_{\Lambda}} \exp \left\{-I_{\Lambda}^{\mathrm{per}}\left(\omega_{\Lambda}\right)\right\} \chi_{\Lambda}\left(\mathrm{d} \omega_{\Lambda}\right),
$$

is the partition function, the same as in (1.9). The corresponding construction can be conducted for the local Gibbs state $\varrho_{\Lambda}$. The Euclidean Gibbs measure $\mu_{\Lambda}$ will 


\section{A. KARGOL, YU. KOZITSKY}

have representation (3.17), (3.19), (3.20) in which the condition $\left|\ell-\ell^{\prime}\right|_{\Lambda}=1$ is replaced by $\left|\ell-\ell^{\prime}\right|=1$.

We conclude this section by giving the path integral representation of the local polarizations and Matsubara functions (2.5). They are

$$
\begin{aligned}
M_{\Lambda}^{\mathrm{per}}(J, h) & =\int_{\Omega_{\Lambda}} \omega_{\ell}(0) \mu_{\Lambda}^{\mathrm{per}}\left(\mathrm{d} \omega_{\Lambda}\right), \\
M_{\Lambda}(J, h) & =\frac{1}{|\Lambda|} \sum_{\ell \in \Lambda} \int_{\Omega_{\Lambda}} \omega_{\ell}(0) \mu_{\Lambda}\left(\mathrm{d} \omega_{\Lambda}\right), \\
\Gamma_{\ell \ell^{\prime}}^{\Lambda}\left(\tau, \tau^{\prime}\right) & =\int_{\Omega_{\Lambda}} \omega_{\ell}(\tau) \omega_{\ell^{\prime}}\left(\tau^{\prime}\right) \mu_{\Lambda}\left(\mathrm{d} \omega_{\Lambda}\right), \\
\Gamma_{\ell \ell^{\prime}}^{\Lambda}\left(\tau, \tau^{\prime} \mid \text { per }\right) & =\int_{\Omega_{\Lambda}} \omega_{\ell}(\tau) \omega_{\ell^{\prime}}\left(\tau^{\prime}\right) \mu_{\Lambda}^{\mathrm{per}}\left(\mathrm{d} \omega_{\Lambda}\right) .
\end{aligned}
$$

\section{COMMENTS AND PROOF OF THE LEMMAS}

\section{A. Comments on Propositions}

Here we discuss the proof of Propositions I.1, II.2, II.3, II.5, II.6, and II.8.

By means of the Euclidean realization of states (2.1) described in the previous section one can prove that the net $\left\{\varrho_{\Lambda}\right\}_{\Lambda \Subset \mathbb{L}}$, as well as the sequence $\left\{\varrho_{\Lambda}^{\text {per }}\right\}_{\Lambda \in \mathcal{L}_{\text {box }}}$, are relatively compact in the topology which guarantees the convergences stated in the propositions under question. The boundedness follows from the moment estimates for Euclidean Gibbs measures proved in [19,27,28]. The equalities in (1.12) can be obtained by means of Theorem 7.12 of [29]. The inequalities (2.6) follow from the FKG inequality, for the Euclidean Gibbs measures proved in $[18,19]$. The proof of $(2.7)$ was performed in [19], see Lemma 6.4. If (2.19) holds, then the limiting periodic Euclidean Gibbs states are nonergodic with respect to the group of translations of $\mathbb{L}$, which certainly means a phase transition, see [19] and the references therein. Finally, estimate (2.30) is the infrared bound, the proof of which is standard, see $[5-7,18,19]$.

\section{B. Proof of Lemma II.9}

We start by proving the first line in (2.32). To this end, we find a strictly increasing function $\phi$ : $\left[h_{+}\left(m_{0}\right),+\infty\right) \rightarrow \mathbb{R}$ such that

$$
p_{\Lambda}^{\mathrm{per}}(J, h) \geq \phi(h) \quad \text { for } h \geq h_{+}\left(m_{0}\right)
$$

Then we use the convexity of $p_{\Lambda}^{\text {per }}(J, \cdot)$ and get the result in question by $(2.2)$. Let us split the potential $V_{0}$ into even and odd parts

$$
V_{0}(x)=V_{0}^{\mathrm{e}}(x)+V_{0}^{\mathrm{o}}(x)
$$

Thereby, for $b>0$, we choose $h>0$ such that, for all $h>\underline{h}, h x_{\ell}-V_{0}^{\mathrm{e}}\left(x_{\ell}\right)$ is an increasing function of $x_{\ell} \in[-b, b]$. Set, c.f. (3.9), (3.17),

$$
\begin{gathered}
\mathcal{C}_{b}=\left\{\left.\omega \in \mathcal{C}|| \omega\right|_{\mathcal{C}} \leq b\right\} \\
\Omega_{\Lambda}^{b}=\left\{\omega_{\Lambda}=\left(\omega_{\ell}\right)_{\ell \in \Lambda} \mid \omega_{\ell} \in \mathcal{C}_{b}, \quad \ell \in \Lambda\right\}, \\
Z_{\Lambda}^{b}(J, h)=\int_{\Omega_{\Lambda}^{b}} \exp \left[-I_{\Lambda}^{\mathrm{per}}\left(\omega_{\Lambda}\right)\right] \chi_{\Lambda}\left(\mathrm{d} \omega_{\Lambda}\right) .
\end{gathered}
$$

Obviously,

$$
p_{\Lambda}^{\text {per }}(J, h) \geq \frac{1}{|\Lambda|} \log Z_{\Lambda}^{b}(J, h) .
$$

By the first GKS inequality, see e.g., Theorem 12.1 in [26],

$$
Z_{\Lambda}^{b}(J, h) \geq Z_{\Lambda}^{b}(0, h) \stackrel{\text { def }}{=} \exp (|\Lambda| \phi(h)), \quad \text { for all } h>\underline{h} .
$$

Here

$$
\exp [\phi(h)]=\int_{\mathcal{C}_{b}} \exp \left\{\int_{0}^{1}\left[h \omega(\tau)-V_{0}(\omega(\tau))\right] \mathrm{d} \tau\right\} \chi(\mathrm{d} \omega) .
$$

By Jensen's inequality, for every $\tilde{h}>0$ and $h \geq \tilde{h}$,

$$
\phi(h) \geq(h-\tilde{h}) \gamma(m, \tilde{h})+\phi(\tilde{h}), \quad \gamma(m, h) \stackrel{\text { def }}{=} \phi^{\prime}(h) .
$$

By (3.14), one can show, see [18], that for any $m_{0}>0$, the family of the corresponding measures $\{\chi\}_{\mid m \geq m_{0}}$ is tight as measures on $\mathcal{C}$. On the other hand, the righthand side of (4.5) can be extended to the whole complex plane as an entire ridge function of $h$ with the ridge being the real axis. Thereby, for any $m_{0}>0$, there should exist $\tilde{h}$ such that

$$
\gamma_{*}(\tilde{h}) \stackrel{\text { def }}{=} \inf _{m \geq m_{0}} \gamma(h, \tilde{h})>0
$$

Then for a fixed $m_{0}$, we take $h_{+}\left(m_{0}\right)=\max \{\tilde{h}, \underline{h}\}$, which yields (4.1) and hence the first part of (2.32). Since we have not employed any property of $V_{0}^{\mathrm{e}}$, the rest of the lemma can be proved by changing the sign of $h$ and all $\omega_{\ell}$.

\section{Main Estimate}

The proof of Lemmas II.10, II.11 is based on the estimate which we derive now.

The path measure $\nu_{h}$ corresponding to the anharmonic oscillator (1.2) with the external field $h$ is defined as a probability measure on $\mathcal{C}$ by the following expression, c. f. (1.3), (3.17)-(3.19), 


$$
\nu_{h}(\mathrm{~d} \omega)=\frac{1}{N_{h}} \exp \left[h \int_{0}^{1} \omega(\tau) \mathrm{d} \tau-\int_{0}^{1} V_{0}(\omega(\tau)) \mathrm{d} \tau\right] \chi(\mathrm{d} \omega)
$$

where

$$
N_{h}=\int_{\mathcal{C}} \exp \left[h \int_{0}^{1} \omega(\tau) \mathrm{d} \tau-\int_{0}^{1} V_{0}(\omega(\tau)) \mathrm{d} \tau\right] \chi(\mathrm{d} \omega) .
$$

Let $h_{ \pm}\left(m_{0}\right)$ be as in Lemma II.9. Then for $\epsilon>0$ and

$$
h \in\left[h_{-}\left(m_{0}\right)-\epsilon, h_{+}\left(m_{0}\right)+\epsilon\right], \quad m>m_{0},
$$

by (3.14) we readily get

$$
\int_{\mathcal{C}}\left[\omega(\tau)-\omega\left(\tau^{\prime}\right)\right]^{2 k} \nu_{h}(d \omega) \leq m^{-k} Q_{k} \cdot\left|\tau-\tau^{\prime}\right|_{\mathrm{per}}^{k}, \quad k \in \mathbb{N},
$$

which holds, uniformly in $h$ and $m$ obeying (4.9), with $Q_{k}$ depending on $\epsilon$ only. In the sequel, we always assume that $h$ and $m$ are chosen according to (4.9).

Since the potential $V_{0}$ is continuous and defined on the whole $\mathbb{R}$, every one-dimensional projection of $\nu_{h}$ is nondegenerate, which yields that for every $n \in \mathbb{N}, \tau_{1}, \ldots, \tau_{n} \in[0,1]$, and $c>0$ both sets

$$
C^{ \pm}\left(\tau_{1}, \ldots, \tau_{n} ; c\right) \stackrel{\text { def }}{=}\left\{\omega \in \mathcal{C} \mid \pm \omega\left(\tau_{j}\right) \geq c, \text { for all } j=1, \ldots, n\right\}
$$

are such that $\nu_{h}\left[C^{ \pm}\left(\tau_{1}, \ldots, \tau_{n} ; c\right)\right]>0$. By $C^{ \pm}(n ; c)$ we denote $C^{ \pm}\left(\tau_{1}, \ldots, \tau_{n} ; c\right)$ with $\tau_{j}=j / n, j=1, \ldots, n$.

Lemma IV.1 For every integer $n \geq 2$ and any $\varepsilon>0$, there exist $\underline{m}>m_{0}, c>\sqrt{\varepsilon}$, and $B_{\varepsilon}^{ \pm} \subset C^{ \pm}(n ; c)$, such that for all $m>\underline{m}$,

$$
\nu_{h}\left(B_{\varepsilon}^{ \pm}\right)>0
$$

and for all $\omega \in B_{\varepsilon}^{ \pm}$,

$$
\forall \tau \in[0,1]: \quad \pm \omega(\tau) \geq \sqrt{\varepsilon} .
$$

Proof: Let us fix $p \in \mathbb{N} \backslash\{1\}, \alpha \in(0, p-1)$, and set

$$
\begin{aligned}
& \lambda_{\vartheta}(\omega)=\sup \left\{\frac{\left[\omega(\tau)-\omega\left(\tau^{\prime}\right)\right]^{2 p}}{\left|\tau-\tau^{\prime}\right|_{\text {per }}^{\alpha}}|0<| \tau-\left.\tau^{\prime}\right|_{\text {per }} \leq \vartheta\right\}, \\
& \vartheta \in(0,1) .
\end{aligned}
$$

Then by the Garsia-Rodemich-Rumsey lemma, see e.g., pages 202, 203 in [31], one has from (4.10)

$$
\begin{aligned}
\int_{\mathcal{C}} \lambda_{\vartheta}(\omega) \nu_{h}(d \omega) & \leq \frac{2^{\alpha+6 p+\varsigma}}{p-\alpha-1}\left(1+\frac{2}{\alpha}\right) m^{-p} Q_{p} \vartheta^{p-\alpha} \\
& \stackrel{\text { def }}{=} m^{-p} Q_{p, \alpha} \vartheta^{p-\alpha}
\end{aligned}
$$

where $\varsigma>0$ is an absolute constant. Now we fix $n \geq 2$ and for $c>\sqrt{\varepsilon}$, define

$$
\begin{aligned}
A(c ; \varepsilon) & =\left\{\omega \in \mathcal{C} \mid \lambda_{1 / n}(\omega) \leq(c-\sqrt{\varepsilon})^{2 p} n^{\alpha}\right\}, \\
B_{\varepsilon}^{ \pm} & =A(c ; \varepsilon) \bigcap C^{ \pm}(n ; c) .
\end{aligned}
$$

Then for any $\tau \in[0,1]$, one can pick up $j / n$, such that

$$
|\omega(\tau)-\omega(j / n)| \leq\left[\lambda_{1 / n}(\omega)\right]^{1 / 2 p} n^{-\alpha / 2 p},
$$

which yields $\pm \omega(\tau) \geq \sqrt{\varepsilon}$ if $\omega \in B_{\varepsilon}^{ \pm}$. To estimate $\nu_{h}\left(B_{\varepsilon}^{ \pm}\right)$ we proceed as follows. By (4.13) and (4.15), and by the Chebyshev inequality

$$
\begin{aligned}
\nu_{h}[\mathcal{C} \backslash A(c ; \varepsilon)] & \leq \frac{1}{(c-\sqrt{\varepsilon})^{2 p} n^{\alpha}} \int \lambda_{1 / n}(\omega) \nu_{h}(\mathrm{~d} \omega) \\
& \leq m^{-p} \cdot Q_{p, \alpha} /\left[n(c-\sqrt{\varepsilon})^{2}\right]^{p} .
\end{aligned}
$$

Now we set

$$
\sigma(n ; c)=\min \left\{\nu_{h}\left[C^{+}(n ; c)\right] ; \nu_{h}\left[C^{-}(n ; c)\right]\right\} .
$$

Thereby,

$$
\begin{aligned}
\nu_{h}\left(B_{\varepsilon}^{ \pm}\right) & \geq \sigma(n ; c)-\nu_{h}[\mathcal{C} \backslash A(c ; \varepsilon)] \\
& \geq \sigma(n ; c)-m^{-p} \cdot Q_{p, \alpha} /\left[n(c-\sqrt{\varepsilon})^{2}\right]^{p},
\end{aligned}
$$

which is positive for all

$m>\underline{m} \stackrel{\text { def }}{=} \max \left\{m_{0} ;\left[n(c-\sqrt{\varepsilon})^{2}\right]^{-1} \cdot\left[Q_{p, \alpha} / \gamma(n ; c)\right]^{1 / p}\right\}$.

Now we introduce

$$
\mathcal{C} \times \mathcal{C} \ni\left(\omega, \omega^{\prime}\right) \mapsto Y\left(\omega, \omega^{\prime}\right)=\int_{0}^{1} \omega(\tau) \omega^{\prime}(\tau) d \tau .
$$

Then, by (4.13)

$$
\forall \omega, \omega^{\prime} \in B_{\varepsilon}^{ \pm}: \quad Y\left(\omega, \omega^{\prime}\right) \geq \varepsilon .
$$

\section{Proof of the Lemmas}

Proof of Lemma II.10: In the Euclidean approach, the periodic pressure $(2.1)$ has the following representation, see (3.20), (3.19), and (4.7), 


$$
p_{\Lambda}^{\mathrm{per}}(J, h)-p_{\Lambda}^{\mathrm{per}}(0, h)=|\Lambda|^{-1} \log \left\{\int_{\Omega_{\Lambda}} \exp \left[J Y_{\Lambda}\left(\omega_{\Lambda}\right)\right] \prod_{\ell \in \Lambda} \nu_{h}\left(d \omega_{\ell}\right)\right\},
$$

where

$$
Y_{\Lambda}\left(\omega_{\Lambda}\right)=\frac{1}{2} \sum_{\ell, \ell^{\prime} \in \Lambda,\left|\ell-\ell^{\prime}\right|_{\Lambda}=1} Y\left(\omega_{\ell}, \omega_{\ell^{\prime}}\right)
$$

and $Y$ being as in (4.19). For $\pm h \geq 0$, we get from (4.21), (4.20)

$$
p_{\Lambda}^{\mathrm{per}}(J, h)-p_{\Lambda}^{\mathrm{per}}(0, h) \geq|\Lambda|^{-1} \log \left\{\int_{\left(B_{\varepsilon}^{ \pm}\right)^{|\Lambda|}} \exp \left[J Y\left(\omega_{\Lambda}\right)\right] \prod_{\ell \in \Lambda} \nu_{h}\left(d \omega_{\ell}\right)\right\} \geq d J \varepsilon+\nu_{h}\left(B_{\varepsilon}^{ \pm}\right) .
$$

Now we fix $\varepsilon, c, n$, and $\alpha$. Then for a given $\delta>0$, we denote by $m_{*}$ the value of $m$ for which the second line in (4.17) equals $\exp (-\delta)$. Thereafter, the latter estimate turns into (2.33).

Proof of Lemma II.11: First we write

$$
p_{\Lambda}^{\text {per }}(J, h)-p_{\Lambda}(0, h)=\int_{0}^{J}\left(\frac{\partial}{\partial t} p_{\Lambda}^{\text {per }}(t, h)\right) d t \leq J \frac{\partial}{\partial J} p_{\Lambda}^{\text {per }}(J, h),
$$

since $p_{\Lambda}^{\text {per }}$ is a convex function of $J$. Then by (4.21), (4.22), (4.19),

$$
\begin{aligned}
\frac{\partial}{\partial J} p_{\Lambda}^{\text {per }}(J, h) & =\frac{1}{2|\Lambda|} \sum_{\ell, \ell^{\prime} \in \Lambda,\left|\ell-\ell^{\prime}\right|_{\Lambda}=1} \int_{\Omega_{\Lambda}} \omega_{\ell}(0) \omega_{\ell^{\prime}}(0) \mu_{\Lambda}^{\text {per }}\left(\mathrm{d} \omega_{\Lambda}\right) \\
& \leq \frac{1}{4|\Lambda|} \sum_{\ell, \ell^{\prime} \in \Lambda,\left|\ell-\ell^{\prime}\right|_{\Lambda}=1} \int_{\Omega_{\Lambda}}\left\{\left[\omega_{\ell}(0)\right]^{2}+\left[\omega_{\ell^{\prime}}(0)\right]^{2}\right\} \mu_{\Lambda}^{\mathrm{per}}\left(\mathrm{d} \omega_{\Lambda}\right) \\
& =\frac{1}{4|\Lambda|} \sum_{\ell, \ell^{\prime} \in \Lambda,\left|\ell-\ell^{\prime}\right|_{\Lambda}=1}\left\{\varrho_{\Lambda}^{\mathrm{per}}\left(q_{\ell}^{2}\right)+\varrho_{\Lambda}^{\mathrm{per}}\left(q_{\ell^{\prime}}^{2}\right)\right\}=d \varrho_{\Lambda}^{\mathrm{per}}\left(q_{\ell}^{2}\right),
\end{aligned}
$$

since $\varrho_{\Lambda}^{\text {per }}\left(q_{\ell}^{2}\right)$ is independent of $\ell$. Here, c.f., (4.21),

$$
\begin{aligned}
\mu_{\Lambda}^{\mathrm{per}}\left(d \omega_{\Lambda}\right) & =\exp \left[F_{\Lambda}(J, h)+J Y_{\Lambda}\left(\omega_{\Lambda}\right)\right] \prod_{\ell \in \Lambda} \nu_{h}\left(d \omega_{\ell}\right), \\
F_{\Lambda}(J, h) & =|\Lambda|\left[p_{\Lambda}(0,0)-p_{\Lambda}^{\mathrm{per}}(J, h)\right]
\end{aligned}
$$

is the Euclidean local Gibbs measure which corresponds to the Hamiltonian (1.8). Thereby, we employ (4.23) in (4.23) and get (2.34).

[1] J. Fröhlich, B. Simon, T. Spencer, Commun. Math. Phys. 50, 79 (1976).

[2] F. J. Dyson, E. H. Lieb, B. Simon, J. Statist. Phys. 18, 335 (1978).

[3] W. Driessler, L. Landau, J. F. Perez, J. Statist. Phys. 20, 123 (1979).

[4] L. A. Pastur, B. A. Khoruzhenko, Teoret. Mat. Fiz. 73, 111 (1987).

[5] V. S. Barbulyak, Y. G. Kondratiev, Rep. Natl. Acad. Sci. Ukr. 10, 19 (1991).

[6] V. S. Barbulyak, Y. G. Kondratiev, Func. Anal. Appl 26(2), 61 (1992).

[7] Ju. G. Kondratiev, in Stochastic Processes, Physics, and Geometry II, (World Scientific, Singapore New Jersey, 1994), p. 465.

[8] S. Albeverio, Y. Kondratiev, Y. Kozitsky, M. Röckner,
Phys. Rev. Lett. 90, 170603 (2003).

[9] S. Albeverio, Y. Kondratiev, Y. Kozitsky, M. Röckner, Ann. Inst. H. Poincaré Probab. Statist. 37, 43 (2001).

[10] S. Albeverio, Y. Kondratiev, Y. Kozitsky, M. Röckner, Commun. Math. Phys. 241, 69 (2003).

[11] S. Albeverio, Y. Kondratiev, Y. Kozitsky, Lett. Math. Phys. 48, 221 (1999).

[12] R. Blinc, B. Žekš, Soft Modes in Ferroelectrics and Antiferroelectrics (North-Holland Publishing Company/Americal Elsevier, Amsterdam London New York, 1974).

[13] S. Stamenković, Condens. Matter Phys. 1(14), 257, (1998).

[14] V. G. Vaks, Introduction to the Microscopic Theory of Ferroelectrics (Nauka, Moscow, in Russian 1973).

[15] B. Simon, The $P(\phi)_{2}$ Euclidean (Quantum) Field Theo- 


\title{
A PHASE TRANSITION IN A QUANTUM ANHARMONIC CRYSTAL
}

$r y$ (Princeton University Press, New York San Francisco London, 1974).

[16] J. Glimm, A. Jaffe, Quantum Physics. A Functional Integral Point of View (Second Edition, Springer-Verlag, New York Berlin Heidelberg London Paris Tokyo, 1987).

[17] O. Bratteli, D. W. Robinson, Operator Algebras and Quantum Statistical Mechanics,I, II (Springer-Verlag, New York, 1981).

[18] S. Albeverio, Y. Kondratiev, Y. Kozitsky, M. Röckner, Rev. Math. Phys. 14, 1, (2002).

[19] Y. Kozitsky, T. Pasurek, J. Statist. Phys. 127, 985 (2007).

[20] A. Klein, L. Landau, J. Funct. Anal. 42, 368, (1981).

[21] Y. Kozitsky, Lett. Math . Phys. 68, 183 (2004).

[22] L. Birke, J. Fröhlich, Rev. Math. Phys. 14, 829 (2002).

[23] R. Gielerak, L. Jakóbczyk, R. Olkiewicz, J. Math. Phys. 35, 3762 (1994).

[24] B. V. Shabat, Introduction to Complex Analysis. II:
Functions of Several Variables (Translations of Mathematical Monographs, 110. American Mathematical Society, Providence, RI. (1992).

[25] B. Simon, The Statistical Mechanics of Lattice Gases, I (Princeton University Press, Princeton, New Jersey, 1993).

[26] B. Simon, Functional Integration and Quantum Physics (Academic Press, New York and London, 1979).

[27] S. Albeverio, Y. G. Kondratiev, T. Pasurek, M. Röckner, Ann. Probab. 32, 153 (2004).

[28] S. Albeverio, Y. G. Kondratiev, T. Pasurek, M. Röckner, Transec. Moscow Math. Society 1, 3 (2005).

[29] H.-O. Georgii, Gibbs Measures and Phase Transitions. (Studies in Mathematics, 9, Walter de Gruyter, Berlin New York, 1988).

[30] A. M. Garsia, E. Rodemich, H. Rumsey, Jr., Indiana Univ. Math. J. 20, 565 (1970/1971).

[31] M. T. Barlow, M. Yor, J. Funct. Anal. 49, 198 (1982).

\section{ФАЗОВИЙ ПЕРЕХІД У КВАНТОВОМУ АНГАРМОНІЧНОМУ КРИСТАЛІ}

\author{
А. Карголь, Ю. Козицький \\ Інститут математики, Університет Марії Кюрі-Склодовсъкої, 20-031, Люблин, Польща
}

\begin{abstract}
Розглянуто трансляційно-інваріянтну систему взаємодіючих квантових ангармонічних осциляторів, індексованих елементами $d$-вимірної простої кубічної гратки. Ангармонічний потенціял є загального типу, що, зокрема, означає відсутність будь-якої симетрії. Для цієї системи ми доводимо, що глобальна поляризація (одержана у термодинамічній границі) стає розривною для деякого значення зовнішнього поля, якщо $d \geq 3$, а також маса частинки й інтенсивність взаємодії є достатньо великими. Доведення зроблено на основі представлення локальних станів Гіббса моделі у вигляді мір на стежках, що дає змогу використати інфрачервоні оцінки та нерівність Гарсії-Родеміха-Рамсея.
\end{abstract}

